\title{
Power system generation reliability assessment based on multi-agents technique
}

\author{
Nadheer A. Shalash*, Abu Zaharin Bin Ahmad \\ Faculty of Electrical and Electronics Engineering, University Malaysia Pahang, Malaysia
}

\begin{abstract}
Recently, Multi-Agent System (MAS) is predicted to play important role in the electric power system in term of fast decision, control and reliability. In this paper, a new application of multi-agent system to present hierarchical level-I (HL-I) for power system generation reliability assessment is proposed. The MAS consist of three agents as follows; first agent is the generator agent which is used to connect the generator to the grid depending on the state of the load demand, the second is a load agent is that located at the load and third is so-called the "master agent" that control the generators and load agents together. The agents are created from IEEE 30-bus test system that implemented by using the JADE package in term of loss of load probability index and loss of load expectation index for each state by Monte Carlo method. The simulation result shows the effectiveness of proposed multi-agent approach to provide fast and cost effective techniques.
\end{abstract}

Keywords: Generation reliability, multi-agent techniques, monte carlo, jade package

\section{Introduction}

Reliability of the generated power system is afflicted with the load curve characteristics, peak duration and variety between levels of the peak at each hour, day and month of each season of a year. Various kinds of customers might have different load curve charts. The most frequent categorization for electrical loads is: residential, commercial and industrial which usually each load curve contains a characteristic chart. Such a process's quality is just a strong task of the dispatcher's understanding of the system topology, utilization of automation, and typical trouble call techniques [1]. Probability based models have already been advanced for precisely reflects the stochastic nature of generators behaviour and determines its reliability interpretation [2]. As a result of the demand of increased powers, a small separated system was not able to provide power with sufficient generation control and an acceptable level of reliability at hierarchical level-I (HL-I) includes the ability of the generation facilities to meet the total system load requirement [3] and to have the reserve margin capacity to serve for planned and forced outage events, in order to be able to accurately meet up with the large fluctuations in the load as a result of different quantity of power users, to decrease overall production costs and maintain through the usage of a diversified systems, personal generating stations and finally the utility companies united with each other over a transmission and distribution network to make larger power pools [4]. Today the power quality and reliability are one of the most crucial features combined with the cost in the power generation.

There are two techniques for evaluating power system reliability: analytical and simulation methods. The simulation methods can be utilized to fix the issue of the distribution evaluation, assess the reliability indices by simulating the particular process and unplanned behaviour of the system as randomly [5]. Monte Carlo simulation (MCS) method is latterly receiving considerable interest as the simulation method [2]. Therefore, a MCS has been employed to approximate the required calculations for generation

\footnotetext{
* Manuscript received May 16, 2013; revised July 20, 2013.

Corresponding author. Tel.: +6-0169795140; E-mail address: eng_n_a_msc@yahoo.com.
} 
reliability assessment when the system is complicated.

Power system reliability can be divided into two aspects, adequacy and security. System adequacy relates to the existence of appropriate generators within the system to cater the system demand or system operational constraints and to have excess capacity to cater for planned and forced outage events. Most widely used indices in the (HL-I) reliability evaluations are: loss of load expectation (LOLE) and loss of load probability (LOLP). These suitable indices can describe generation reliability levels when the load is more than the available generation and determination of required reserves.

Nowadays, agents are the focus of intense attention in many areas such as computer science and artificial intelligence. In facts, agents are being used in an increasingly wide variety of applications [6]. A multi-agent system is a system composed of multiple interacting intelligent agents and wide used in other branches of engineering and computer science [7]. For the distributed approach using the multi-agent approach for solving power system problems, the power system network can be decomposed by dividing the system into subsystems with respect to the voltage levels [8]. For consistent service to the electricity consumers, power system must remain intact and be able to endure a wide variety of disturbances. It should be operated in normal and secured condition. As a result, the system must be designed and operated so that the more possible contingencies can be sustained with no loss of load [9]. In order to efficiently manage and control the power network, multi-agent systems (MASs) have been employed to solve the challenges in power networks, and are being developed for a range of applications including fault diagnostics, system monitoring, system restoration, system simulation, and system control [10].

We are able to design the MCSs as one agent that plays repetitively against itself by utilizing a play out approach [11]. As JADE (java agent development framework) is written totally in Java [12]. JADE is a software package to easily create the development of multi-agent techniques in compliance with the foundation for intelligent physical agents (FIPA) descriptions [13]. Many classes expand the size of the reliability table stored and kept by an agent, so expanding memory and computing necessities [14].

The Monte Carlo has been defined as one of the stochastic techniques [2], [5], that is based on the use of random numbers and the calculations the possibilities for investigating the problems. But it takes longer time in calculations and more frequent operation possibilities, to minimize these disadvantages this paper is focused on the application of a multi-agent approach to generation reliability assessment of power system. The proposed MAS for (HL-I) reliability evaluation, based on LOLE and LOLP for adequacy system. The proposed technique is tested using IEEE 30-bus test system and compared with Monte Carlo methods by using JADE package. The simulation results show the effectiveness of proposed multi-agent approach to provide fast and cost effective technique.

\section{The Concept of Reliability Evaluation}

For reliable system, the installed capacity in power system must be higher than expected consumption. A reserve power needs to be provided for frequency regulation and in case of major aggregate loss of capacity. Generation reserve margin method is a measure that shows how the capacity of the power system exceeds the peak consumption and this reserve is calculated by [15],

$$
G_{r m}(\%)=\left(\frac{C_{s}-L_{p}}{C_{s}}\right) \times 100
$$

where $C_{s}$ is the capacity in service and $L_{P}$ is the the total system load demand.

The percent reserve evaluation is calculated by comparing the total installed generating capacity at peak with the total system load. Previous experiments advised to stay reserve margins in the range of 15$25 \%$ of the total system load demand [15]. The reliability expresses the proportion time of the component if it is "in service" or "available for". However, the availability (A) of a component is expressed as [16],

$$
A=\frac{\sum(\text { uptime })}{\sum(\text { uptime })+\sum(\text { downtime })}
$$


where the "up time" is the total time when the component is in service and "down time" is the total time when component not in service. Availability may be expressed in terms of mean time to failure (MTTF) and mean time to repair (MTTR) as given by,

$$
A=\frac{M T T F}{M T T F+M T T R}
$$

The mean time between failures (MTBF) is the sum of MTTF and MTTR and can be represented by cycle of time " $T$ " as seen in Fig. 1.

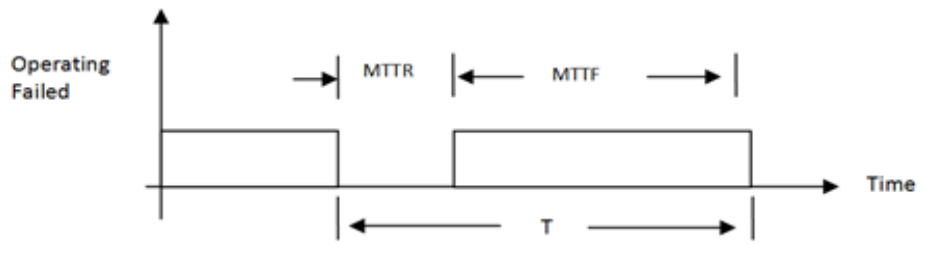

Fig. 1. Mean time diagram for a two state component.

Power system components can be characterized by discrete system states with constant transition rates between these states. State "0" represents the healthy component in an operating condition. As for state "1" (failed state) when the component cannot perform its intended function. Transition occurs between state " 0 " and " 1 " and the transition rates between these states are the failure rate " $\lambda$ " and the repair rate " $\mu$ " as shown in Fig. 2. Therefore, Eq. (2) may be re-expressed in terms of failure rate and repair rate such that

$$
A=\frac{\mu}{\mu+\lambda}
$$

Fig. 2. State space diagram of transition rates.

The unavailability (Q) is sometimes known as the forced outage rate (FOR), its converse of the availability and can be defined in similar terms as in Eq. (5) or in terms of failure rate and repair rate as in Eq. (6).

$$
Q=\frac{\sum \text { downtime }}{\sum \text { uptime }+\sum \text { downtime }}
$$

Therefore, Eq. (2) may be re-expressed in terms of failure rate and repair rate such that,

$$
Q=\frac{\lambda}{\lambda+\mu}
$$

The unit unavailability is a good approximation of a unit failure probability even when preventive maintenance is considered, provided that maintenance is scheduled during low demand periods. Normally, the failure and repair rates are not constant, but may be a function of time such as the Normal, Lognormal, Exponential, Gamma, Weibull, Binomial, and Poisson. In this work, binomial distribution method (BDM) is used [17]. The binomial distribution can be represented by,

$$
(A+Q)^{n}
$$

Eq. (7) [18] can be applicable if the fixed number of trials " $n$ " is known and has resulted in either a 
success or a failure with corresponding probabilities A and Q respectively, all trials must be independent and have identical probabilities of success and therefore fail.

The basic probability principles and combining the different generating units are used for calculating the LOLP. The success probability or availability and its complement, i.e., failure probability or unavailability of each generating unit is the input data. All combinations of available and unavailable generating units are presented in tabular form together with the calculated system availability.

Most of the basic indices used in the generating system adequacy evaluation are expected value of random variables (BDM). Among these indices are; A LOLP and LOLE. LOLP is method tests the probabilities of simultaneous outages of generating capacity that together with a model of daily peak-hour loads determine the number of days per year of expected capacity shortages by,

$$
L O L P=\sum_{i=1}^{n} p_{i} t_{i}
$$

where $p_{i}$ is the probability of system state $i$ and $t_{i}$ is the time interval of capacity in outage

The LOLE index gives the expected (or mean) number of days or hours (days/year or hours/year) in a given period (usually on year) in which the daily peak load or hourly load exceeds the available generating capacity as,

$$
L O L E=\sum_{i \in s} p_{i} T
$$

where $T$ is the time unit of the index (i.e. either one day or one hour ) and $S$ is a set of all possible system states associated with loss of load. The term LOLP is closely related to the term LOLE, the loss of load expectation can be obtained using the daily peak load variation curve.

\section{Multi Agent System (MAS) by Jade Package.}

The multi agent system (MAS) has been extensively used in the evaluation of the difficult problems of in the industry and system reconfiguring. In addition, multi-agent can be used in many field systems composed of multiple autonomous components demonstrating the following features; 1) each agent has incomplete in terms of global control and capabilities for solving problems, 2) The data are decentralized and computation is asynchronous and 3) should be designed according to the problems under consideration [19]. An agent is a physical or virtual entity that essentially has the following properties;

- Agents live and act in a given environment.

- Agents are able to sense its local environment and to interact with other agents in its local environment.

- Agents attempt to achieve particular goals or perform particular tasks.

- Agents are able to respond in a timely fashion to change any occurs in them based on their learning ability.

In general, MAS is a computational system in which several agents interact or work together to achieve goals or perform particular tasks. So the four following elements should be defined when MAS is used to solve problems [20]:

- The meaning and the purpose of each agent in MAS

- An environment where all agents live.

- The definition of a local environment.

- A set of behavioural rules, governing the interaction between the agents and their environment. They are the laws of the agent universe.

JADE (java agent development environment) is typically the most famous representative middleware which accessories an agent program and a development package. JADE emerged in 2000 by the Research and Development Department of Telecom Italia [21]. JADE gives the development of unparalleled software agents that can perform a control task, and helps decentralize control architectures [22]. Developing JADE agents are, by developing a Java core class that runs and applying the setup method. This approach is where in actuality the agents are inputted. The supposed efficiency of an agent is applied 
through its behaviours, generally can be found in the message moving stages the get agent identifier approach to the agent class retrieves the local recognition of the specific agent. Agent communication language is the most crucial function in MAS, and contacting send approach, messages could be delivered to different agents easily. The receiving agents may get the message and information by contacting receives method. All the messages are certified with foundation for intelligent physical agents-standards for implementation of intelligent agents (FIPA) criteria by using FIPA agent management. The initialization procedures and the output of JADE run-time environment is shown in Fig. 3.

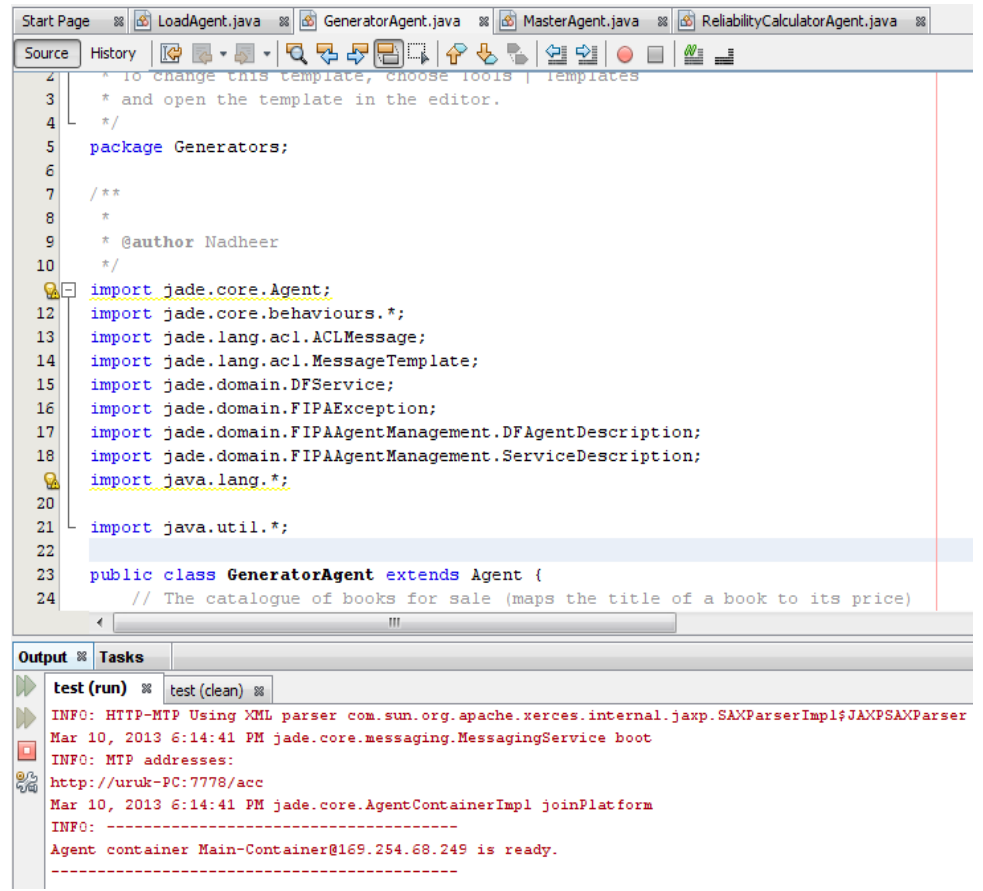

Fig. 3. Initialization procedures and the output of JADE run-time environment.

\section{Proposed Method}

Fig. 4 shows the proposed multi agent can be developed for generator reliability assessment (HLI). Agents will be constructed in three areas, and two levels of decision making and action. In the first area, it has a generator bus called a generator agent, the second area, it has a load bus called a load agent which is attributed to time changes with load, Both that agents are located at the upper level, and in third areas, it has one agent for Monte Carlo methods to calculate the reliability indices that are located at the lower level. Between the two levels has created a master agent implemented on the local area management system.

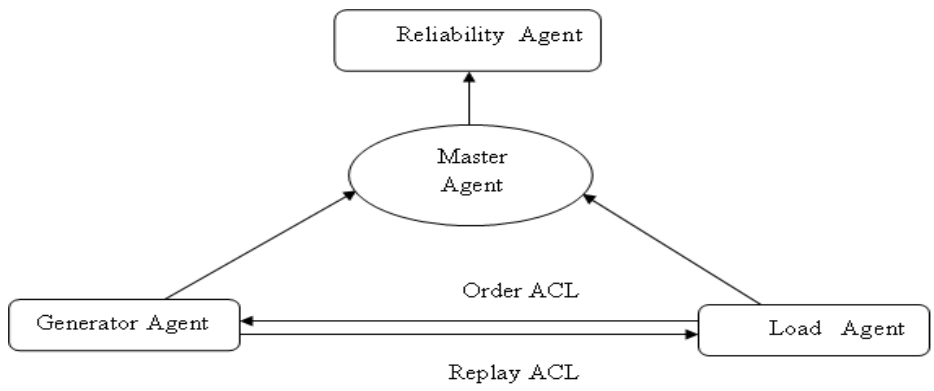

Fig. 4. Agent-based demand management. 
ACL in Fig. 4 is a messageing class, responsible for sending and receiving the information between the agents in JADE package. Here the load agent has order load send it by ACL sending and generator agent replay load agent about available capacity generation by ACL receiving. The introduced agents are detailed as follows;

- Generator agent (GA): The purpose of GA is to decide (connect or not) the connection of the generator to a grid depends on the state of the system. This agent can communicate with other agents for making a decision (refuse/accept). It also has specifications for all generators that send by messages to master agent.

- Load agent (LA): The purpose of LA is to decide whether to shed the load or not depending on a load curve of the area or system. It is done by reading the data from the load and controls it by sending the data message to mastering agent to change the load with time. This due to sometimes the grid has much same size of generator. Master agent takes data from request data message from the load agent and makes a decision (refuse/accept) to generator agent.

- Master agent (MA): The purpose of the MA is to make connections between two principal stages. The first stage reads the data from the agents of generators and loads, where it works on the order of loads on time and sends them to demand generators with a capacity reserve. Second stage sends the resulting data message from the first stage to the agent reliability (RA) depending on the period as indexes to facilitate the reliability of electric power system.

- Reliability agent (RA): The purpose of RA is to calculate reliability indices by Monte Carlo method; it has attributed for probability $(\mathrm{P})$. This agent can communicate with master agent to take past readings of probability between LA and GA to increase the accuracy of predictions.

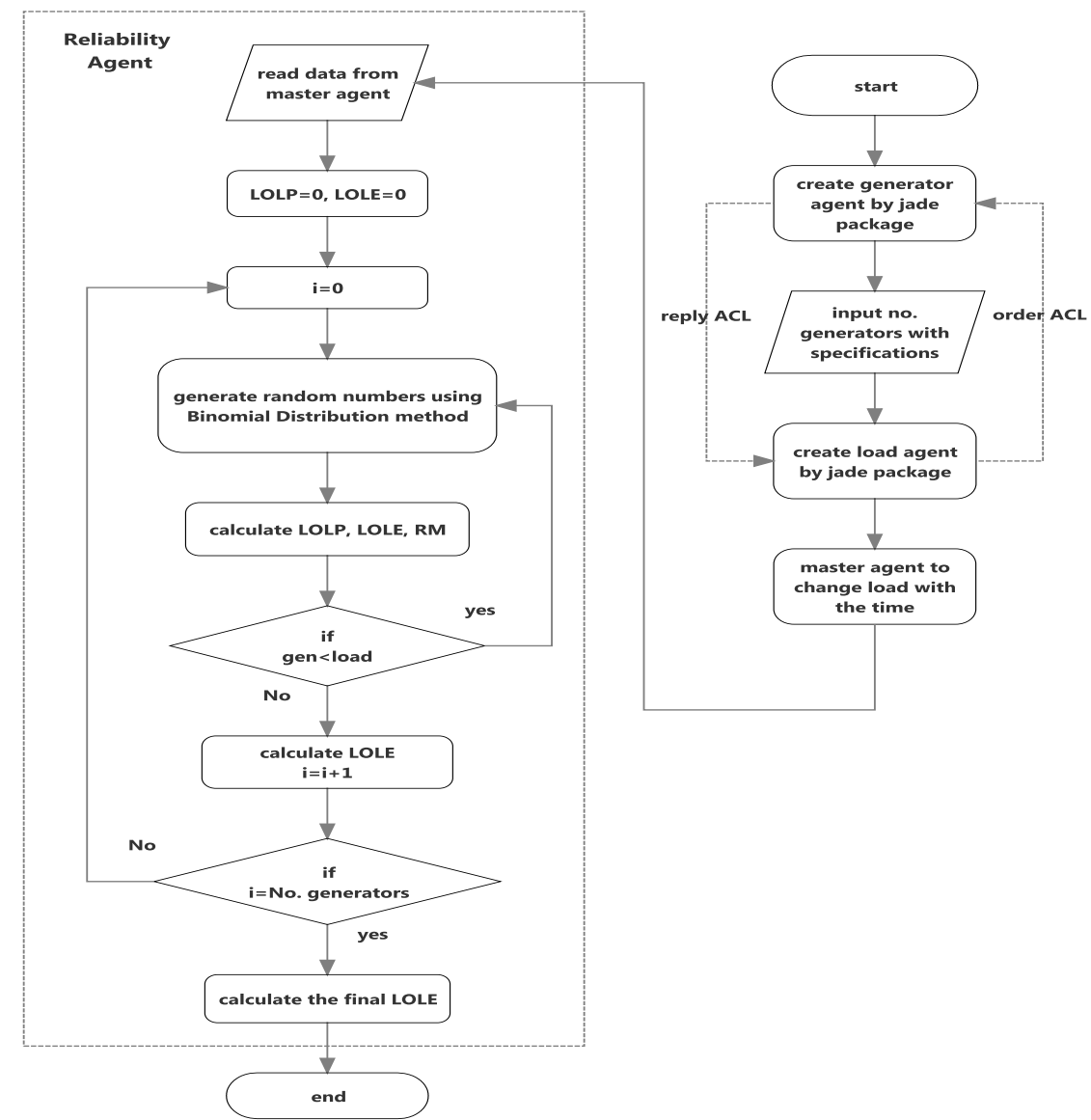

Fig. 5. A Monte Carlo simulation based on multi-agent technique. 
Fig. 5 shows MAS techniques for reliability assessment to measure the response of reliability indices by the following procedures:

1. Create GA, LA and MA by JADE package.

2. Send messages to GA from LA to limit the size and number of generators in service.

3. Take another load by MA.

4. Send all information to RA in order to start calculating reliability indices by MCS..

5. For each selected generators by GA in the previous step, a random number from " 0 " to " 1 " is generated by BDM.

6. Calculate the value of reserve margin and then the sum of the available generators (generations are calculated with the LOLP.

7. A comparison is made between the sum of the generators available to load, if the sum is less than load, the LOLE is calculated.

8. Go to the next iteration.

\section{Result and Discussion}

This part is to demonstrate the effectiveness of the multi agent technique JADE [23] compatible with the FIPA standards [24]. All MAS system information, resources and control can be fully distributed among real hardware terminals in power system. Each generator, loaded in the power system, is considered as stand-alone and independent agent with its own behavior by providing an asynchronous message transferring in a multi-agent system. When some agent does not perform an action (send/receive messages and make computations after refuse/accept messages), it then stops the operation until a new message is sent to the agent [23]. Fig. 6 shows the IEEE 30-bus test system which consist of 6 generator bus with 6 generating units located at $(1,2,13,22,23,27)$ buses, 24 load buses, 41 transmissions. The data of generating units are given in Table 1 . The bus load and injection data are calculated based on the demand on the load buses as shown Table 2 .

Table 1. The data of generating units

\begin{tabular}{ccccccccccc}
\hline \hline $\begin{array}{l}\text { Unit } \\
\text { No. }\end{array}$ & $\begin{array}{c}\text { Bus } \\
\text { No. }\end{array}$ & $\begin{array}{c}\text { Cost coefficient } \\
\text { (\$/MWh) }\end{array}$ & $\begin{array}{c}\text { Pmax } \\
(\mathrm{MW})\end{array}$ & $\begin{array}{c}\text { Pmin } \\
(\mathrm{MW})\end{array}$ & $\begin{array}{c}\text { Min up } \\
\text { time(h) }\end{array}$ & $\begin{array}{c}\text { Min down } \\
\text { time(h) }\end{array}$ & $\begin{array}{c}\text { Ramp up } \\
(\mathrm{MW})\end{array}$ & $\begin{array}{c}\text { Ramp Down } \\
\text { (MW) }\end{array}$ & $\begin{array}{c}\text { Startup ramp } \\
(\mathrm{MW})\end{array}$ & $\begin{array}{c}\text { Shutdown } \\
\text { ramp (MW) }\end{array}$ \\
\hline 1 & 1 & 15 & 80 & 15 & 2 & 2 & 25 & 25 & 70 & 60 \\
2 & 2 & 14.75 & 80 & 15 & 2 & 2 & 25 & 25 & 70 & 60 \\
3 & 13 & 16 & 50 & 10 & 4 & 4 & 15 & 15 & 70 & 60 \\
4 & 22 & 14 & 50 & 10 & 4 & 4 & 15 & 15 & 70 & 60 \\
5 & 23 & 16 & 30 & 5 & 3 & 3 & 10 & 10 & 70 & 60 \\
6 & 27 & 15.25 & 55 & 10 & 4 & 4 & 15 & 15 & 70 & 60 \\
\hline \hline
\end{tabular}

Table 2. Bus load and injection data of IEEE 30-bus test system

\begin{tabular}{cccccc}
\hline \hline Bus No. & Load (MW) & Bus No. & Load (MW) & Bus No. & Load (MW) \\
\hline 1 & 0.0 & 11 & 0.0 & 21 & 17.5 \\
2 & 21.7 & 12 & 11.2 & 22 & 0.0 \\
3 & 2.4 & 13 & 0.0 & 23 & 3.2 \\
4 & 67.6 & 14 & 6.2 & 24 & 8.7 \\
5 & 34.2 & 15 & 8.2 & 25 & 0.0 \\
6 & 0.0 & 16 & 3.5 & 26 & 3.5 \\
7 & 22.8 & 17 & 9 & 27 & 0.0 \\
8 & 30 & 18 & 3.2 & 28 & 0.0 \\
9 & 0.0 & 19 & 9.5 & 29 & 2.4 \\
10 & 5.8 & 20 & 2.2 & 30 & 10.6 \\
\hline \hline
\end{tabular}




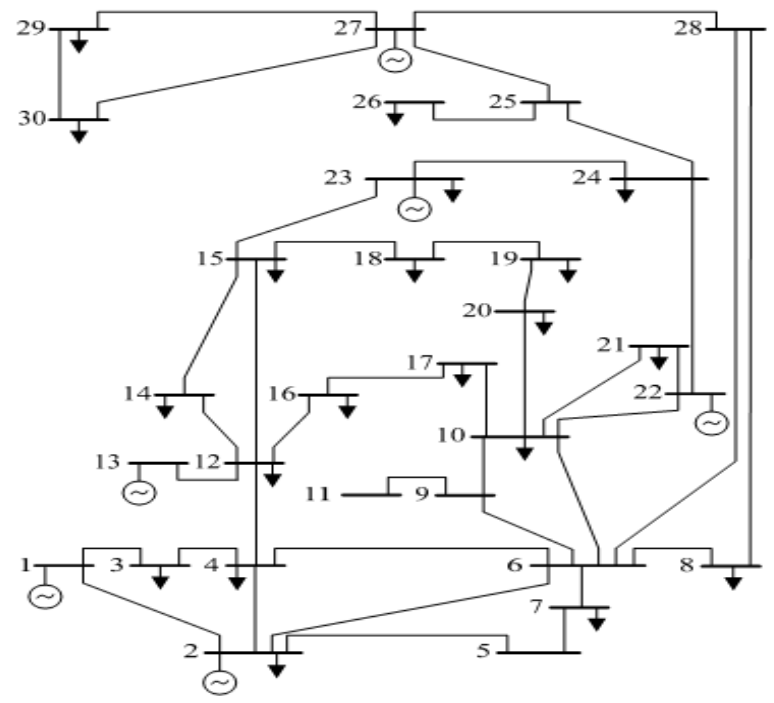

Fig. 6. Single line diagram of the IEEE 30-bus test system.

There will be a number of probabilities to supply the load demand from the available generating capacity. On the other hand, many probabilities are expected to limit the capacity or the number of generating units in service. If the system has a reserve margin or fed the load from the other generator at other bus with low cost, the number of probabilities will be increased which means that the loads can be supplied by all generators and the reliability of the power system will be improved. For example, if The total demanded load is 97.6 MW, where the load in Bus 4 is $67.6 \mathrm{MW}$ and the load in Bus 8 is $30 \mathrm{MW}$, the injected generation into the system can be as follows:

Gen (Bus2):80, Gen (Bus27):30

Gen (Bus1):80, Gen (Bus27):30

Gen (Bus13):50, Gen (Bus22):50

Gen (Bus13):50, Gen (Bus23):55

Gen (Bus22):50, Gen (Bus23):55
Capacity in service $=110$

Capacity in service $=110$

Capacity in service $=100$

Capacity in service $=105$

Capacity in service $=105$

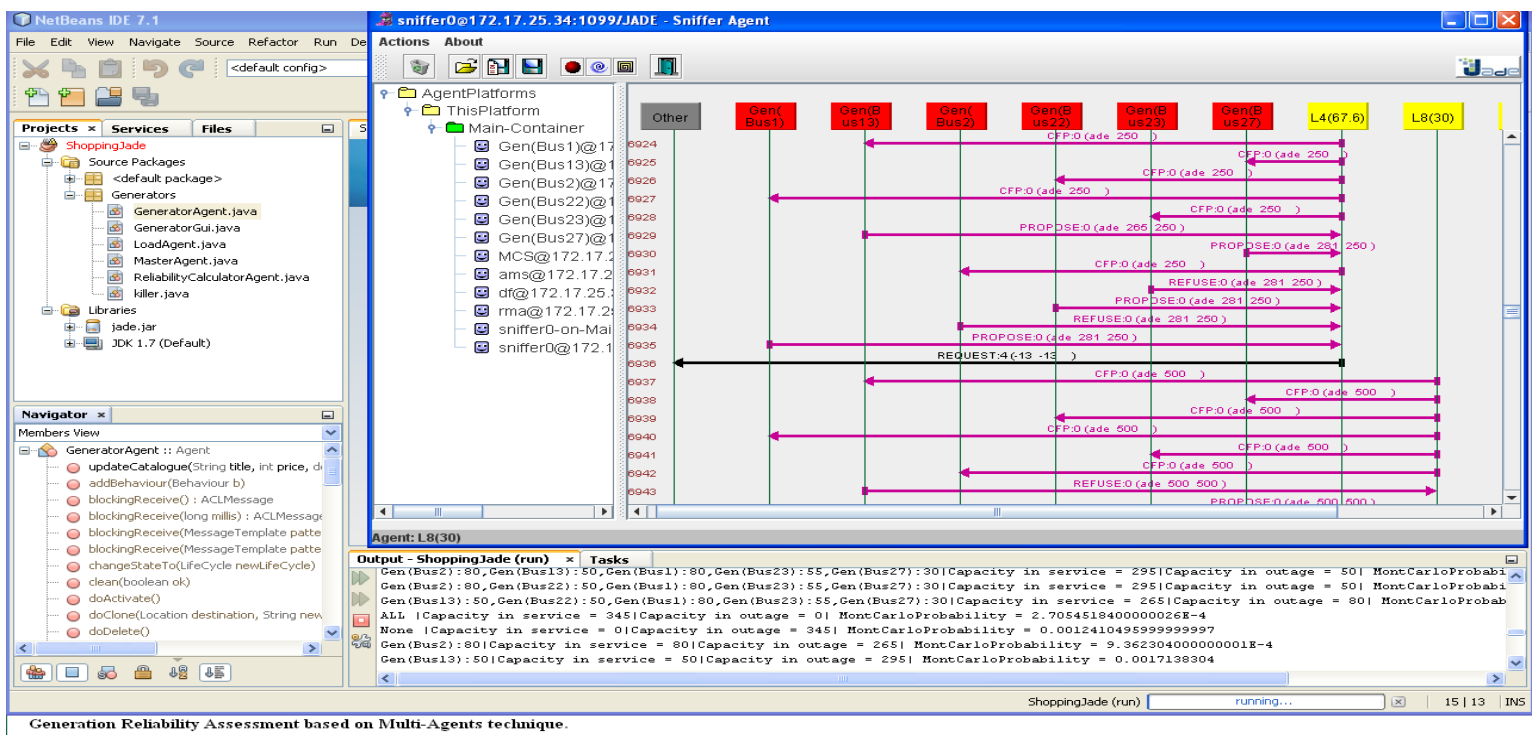

Fig. 7. Virtualization of multi-agent for Load4 (67.6MW) and Load8 (30MW). 
This is confirmed from agent perform some actions (send/receive) messages, make computations of total load as presented in Fig 7. All the communication between load agent and generator agent and calculate all equipment reliability indices for generators under Load4 (67.6MW) and Load8 (30MW).

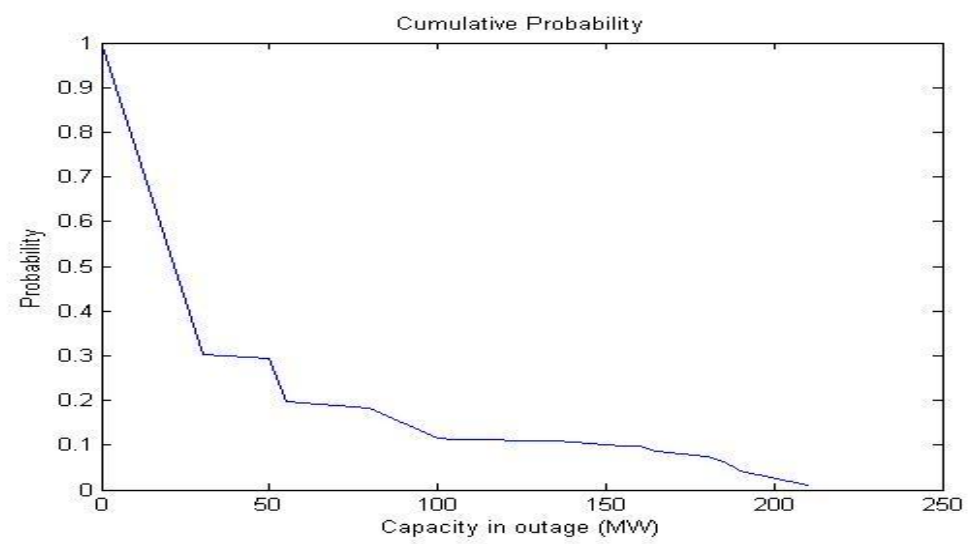

Fig. 8. Distribution of the probability for the IEEE 30-bus test system by (BDM).

The program starts from the normal system state in which all the generating units are in the system is available. Then examine the generation of random figures $[0,1]$ that is necessary for Monte Carlo simulation if the generated number is more than an unavailability of the generator, the generator will be considered available in the mentioned iteration. The fundamental necessity for figures to be random is that each and every figure should have an equal probability of choosing any one of the possible sizes of generator to fed load. Thus, random figures $\mathrm{s}$ in a certain range follows a binomial distribution method (BDM). The random figures applied are highlighted by a computer program and are shown in Table 3. We have many probabilities values are expected to limit the capacity or the number of generating units in service according to Fig 8. For example in Table 3, when the total load is supplied by three units "30 MW $+50 \mathrm{MW}+80 \mathrm{MW}=160 \mathrm{MW}$ ". In this case, four probabilities can be expected to feed 160MW as follows:

Gen (Bus2):80, Gen (Bus13):50, Gen (Bus27):30

Gen (Bus2):80, Gen (Bus22):50, Gen (Bus27):30

Gen (Bus13):50, Gen (Bus1):80, Gen (Bus27):30

Gen (Bus22):50, Gen (Bus1):80, Gen (Bus27):30

Table 3. System evaluation based on probability LOLE and LOLP

\begin{tabular}{ccccc}
\hline \hline $\begin{array}{c}\text { Load } \\
(\mathrm{MW})\end{array}$ & $\begin{array}{c}\text { Time interval of } \\
\text { capacity (Minute) }\end{array}$ & $\begin{array}{c}\text { Capacity of generators in } \\
\text { service }\end{array}$ & LOLP & LOLE \\
\hline 30 & 5 & 30 & $2.822 \mathrm{E}-9$ & 2.0332 \\
50 & 10 & 50 & $3.168 \mathrm{E}-8$ & 1.148 \\
55 & 15 & 55 & $1.568 \mathrm{E}-8$ & 0.9871 \\
80 & 20 & 80 & $7.681 \mathrm{E}-9$ & 0.8245 \\
& & $(50+30)$ & $2.851 \mathrm{E}-7$ & 0.345 \\
100 & 25 & $(50+50)$ & $3.142 \mathrm{E}-6$ & 0.125 \\
130 & 30 & $(80+50)$ & $7.603 \mathrm{E}-7$ & 0.0469 \\
& & $(50+50+30)$ & $2.822 \mathrm{E}-5$ & 0.0143 \\
160 & 35 & $(80+80)$ & $1.843 \mathrm{E}-7$ & 0.0194 \\
& & $(50+50+50+80)$ & $6.843 \mathrm{E}-6$ & 0.0082 \\
185 & 40 & $(80+50+55)$ & 0.001383 & $2.48 \mathrm{E}-4$ \\
& & $(80+80+30)$ & $3.726 \mathrm{E}-5$ & $1.23 \mathrm{E}-3$ \\
215 & 45 & $(80+50+50+80+30)$ & $3.353 \mathrm{E}-4$ & $3.84 \mathrm{E}-5$ \\
& & $(80+80+50+30+50+55)$ & $9.032 \mathrm{E}-6$ & $5.23 \mathrm{E}-5$ \\
240 & 50 & $(80+50+50+30+55+80)$ & $1.642 \mathrm{E}-4$ & $7.05 \mathrm{E}-4$ \\
265 & 55 & & 0.0332 & $6.62 \mathrm{E}-4$ \\
295 & 60 & & 0.0081 & $6.65 \mathrm{E}-6$ \\
345 & 65 & & 0.79668 & $3.201 \mathrm{E}-8$ \\
\hline \hline
\end{tabular}


But one probabilities of them are repeated in each step due to the fact that the two generator groups are having same capacity, these probabilities of selected generators, which is caused by a selection of different sizes of generators data from a set of possible failure data. So to choose the best states then decrease time to calculate the indices of reliability used the reliability agent RA have communicated with the master agent to take readings olden of probability between LA and GA for feeding load.

Table 3, shows the LOLE and LOLP reliability index for all generators under different load condition. Here, the higher value of loss of load expectation indicates that the reliability of the system is low. Whereas the low value of loss of load expectation indicates that the reliability of the system is high. The calculated values of indices in different time intervals will give guidelines for the weakest buses in a power network.

\section{Conclusions}

The multi-agent system was capable to help us make decisions on our items to improve the power system. This paper presents simulation results that show a promising effectiveness of the multi-agent to generation reliability (HL-I). Multi-agent system framework is able to assist the decision making for probability in feeding the total system load requirement. If the load demand is distributed on the capacity of identical generators with small size, the value of reliability indices will decrease and therefore the reliability of the power system will improve. This simulation can be used to decrease the interation and chose the best states then decrease time to calculate the indices of reliability. The proposed multi-agent framework has been identified as a feasible solution to tackle the complexity of modern power systems under various requirements.

\section{References}

[1] Yakowitz SJ. Computational Probability and Simulation. 3rd ed. London: Addison-Wesley Publishing Company; 1989.

[2] Brown RE. Electric Power Distribution Reliability. 2nd ed. New York: Marcel Dekker; 2002.

[3] Wangdee W. Bulk electric system reliability simulation and application. PhD thesis. Department of Electrical Engineering, University of Saskatchewan, Canada S7N 5A9; 2006.

[4] Chaturvedi DK. Soft Computing Techniques and its Applications in Electrical Engineering. Heidelberg: Springer-Verlag Berlin; 2008.

[5] Haroonabadi H, Haghifam MR. Generation reliability assessment in power markets using monte carlo simulation and intelligent systems. World Applied Sciences Journal, 2008; 5(3):302-310.

[6] Nagata T, Watanabe H, Ohno M, Sasaki H. A multi-agent approach to power system restoration. IEEE Transactions on Power Systems, 2002; 17(2):1551-1556.

[7] Liu J, Tang YY, Cao YC. An evolutionary autonomous agents approach to image feature extraction. IEEE Trans. Evol. Comput., 1997; 1(2):141-158.

[8] Panasetsky DA, Etingov PV, Voropai NI. Multi-agent approach to emergency control of power system. In: Proc. of Third International Conference on Electric Utility Deregulation and Restructuring and Power Technologies, 2008:2157-2161.

[9] Oonsivilai A, Greyson KA. Power system contingency analysis using multiagent systems. World Academy of Science, Engineering \& Technology, 2009; 60:355.

[10] Kalinowski B, Anders G. A new look at component maintenance practices and their effect on customer, station and system reliability. Electrical Power \& Energy Systems, 2006; 28(10):679-695.

[11] Marcolino LS, Matsubara H. Multi-agent Monte Carlo Go. Presented at: The International Conference on Autonomous Agents and Multiagent Systems, 2011.

[12] Khatua S, Zhang Y, Das A, Iyengar NChSN. A multi agent based e-shopping system. Journal of Global Research in Computer Science, 2011; 2(4):44-54.

[13] Bellifemine F, Poggi A, Rimassa G. Developing Multi-Agent Systems with JADE. England: John Wiley \& Sons Ltd; 2007.

[14] Tsatsoulis C, Ye G. Learning reliability models of other agents in a multiagent system. Technical report. WS-96-04, 1996. AAAI (www.aaai.org).

[15] Cepin M. Assessment of Power System Reliability Methods And Applications. Springer-Verlag; 2011.

[16] Rios M, Bell K, Kirschen D, Allan R. Computation of the value of security. Research report. Manchester Center for Electrical Engineering. 1999. 
[17] Prada JF. The value of reliability in power systems-pricing operating reserves. Energy Laboratory, Massachusetts Institute of Technology; 1999.

[18] Ash RB. Basic Probability Theory. New York: Dover Publications; 2008.

[19] Zhong W, Liu J, Xue M, Jiao L. Global numerical optimization using multi-agent genetic algorithm. Presented at: The IEEE Fifth International Conference on Computational Intelligence and Multimedia Application, 2003.

[20] Wooldridge M. An Introduction to Multi Agent System. New York: Wiley; 2002.

[21] Chouhan S. Agent based modeling of power distribution systems. Thesis, College of Engineering and Mineral Resources, West Virginia University; 2009.

[22] Colson CM, Nehrir MH, Gunderson RW. Distributed multi-agent microgrids: A decentralized approach to resilient power system self-healing. In: Proc. of IEEE 4th International Conference on Resilient Control Systems (ISRCS), 2011:83-88.

[23] Bellifemine F, Caire G, Trucco T, Rimassa G. JADE programmer's guide. CSELT \& University of Parma, 2000. [Online]. Available: http://jade.tilab.com/doc.

[24] FIPA.Web Site. [Online]. Available: http://www.fipa.org. 